\title{
DEEP IMAGING OF ERIDANUS II AND ITS LONE STAR CLUSTER*
}

\author{
D. Crnojević ${ }^{1}$, D. J. Sand ${ }^{1}$, D. Zaritsky ${ }^{2}$, K. SpekKens $^{3}$, B. Willman ${ }^{2,4,5}$, And J. R. Hargis ${ }^{6}$ \\ ${ }^{1}$ Department of Physics, Texas Tech University, Box 41051, Lubbock, TX 79409-1051, USA; denija.crnojevic@ttu.edu \\ ${ }^{2}$ Steward Observatory, University of Arizona, 933 North Cherry Avenue, Tucson, AZ 85721, USA \\ ${ }^{3}$ Department of Physics, Royal Military College of Canada, Box 17000, Station Forces, Kingston, ON K7L 7B4, Canada \\ ${ }^{4}$ LSST, University of Arizona, 933 North Cherry Avenue, Tucson, AZ 85721, USA \\ 5 Departments of Physics and Astronomy, Haverford College, 370 Lancaster Avenue, Haverford, PA 19041, USA \\ ${ }^{6}$ Space Telescope Science Institute, 3700 San Martin Drive, Baltimore, MD 21218, USA \\ Received 2016 April 27; revised 2016 May 22; accepted 2016 May 27; published 2016 June 9
}

\begin{abstract}
We present deep imaging of the most distant dwarf discovered by the Dark Energy Survey, Eridanus II (Eri II). Our Magellan/Megacam stellar photometry reaches $\sim 3$ mag deeper than previous work and allows us to confirm the presence of a stellar cluster whose position is consistent with Eri II's center. This makes Eri II, at $M_{V}=-7.1$, the least luminous galaxy known to host a (possibly central) cluster. The cluster is partially resolved, and at $M_{V}=-3.5$ it accounts for $\sim 4 \%$ of Eri II's luminosity. We derive updated structural parameters for Eri II, which has a half-light radius of $\sim 280 \mathrm{pc}$ and is elongated $(\epsilon \sim 0.48)$ at a measured distance of $D \sim 370 \mathrm{kpc}$. The colormagnitude diagram displays a blue, extended horizontal branch, as well as a less populated red horizontal branch. A central concentration of stars brighter than the old main-sequence turnoff hints at a possible intermediate-age ( $\sim 3 \mathrm{Gyr}$ ) population; alternatively, these sources could be blue straggler stars. A deep Green Bank Telescope observation of Eri II reveals no associated atomic gas.
\end{abstract}

Key words: galaxies: dwarf - galaxies: individual (Eridanus II) - galaxies: photometry - galaxies: stellar content

\section{INTRODUCTION}

The last several years have seen another burst of discovery of Milky Way (MW) satellites from ATLAS (Belokurov et al. 2014; Torrealba et al. 2016), the Panoramic Survey Telescope \& Rapid Response System (Pan-STARRS; Laevens et al. 2015a, 2015b), the Dark Energy Survey (DES; Bechtol et al. 2015; Drlica-Wagner et al. 2015; Koposov et al. 2015), and the Dark Energy Camera more generally (Kim et al. 2015; Kim \& Jerjen 2015; Martin et al. 2015).

The most distant of the new satellites is Eridanus II (Eri II), at $D \sim 350 \mathrm{kpc}$ and $M_{V} \sim-7 \mathrm{mag}$, which was discovered simultaneously by two groups (Bechtol et al. 2015; Koposov et al. 2015). The distance to Eri II roughly corresponds to the virial radius of the MW ( 300 kpc; e.g., Klypin et al. 2002): this places Eri II near the transition radius between those dwarf spheroidals (with $D \lesssim 300 \mathrm{kpc}$ ), which appear to have lost their primordial gas, and more distant dwarfs which generally retain HI gas reservoirs (e.g., Spekkens et al. 2014). The properties of Eri II suggest that it may be similar to Leo T, another recently discovered dwarf just beyond the virial radius of the MW ( $D=410 \mathrm{kpc}, M_{V} \sim-8$; de Jong et al. 2008), which displays a significant HI gas reservoir (Ryan-Weber et al. 2008) and has undergone multiple epochs of star formation (de Jong et al. 2008; Weisz et al. 2012). Additionally, Koposov et al. (2015) tentatively suggested that Eri II may have a globular cluster, which would make it the smallest known galaxy with its own star cluster.

Motivated by the hints that set Eri II apart from the bulk of the new MW satellites, we obtained deep photometric and HI observations to further study its physical properties. In Section 2, we describe the optical Magellan/Megacam observations, as well as our stellar photometry. In Section 3, we present the color-magnitude diagram (CMD) of Eri II and

\footnotetext{
This paper includes data gathered with the $6.5 \mathrm{~m}$ Magellan Telescopes at Las Campanas Observatory, Chile.
}

its stellar populations, we derive updated values for its distance, structural parameters, luminosity, and HI gas content, and we describe the properties of its stellar cluster. We discuss and conclude in Section 4.

\section{DATA REDUCTION AND PHOTOMETRY}

Eri II was observed on 2015 October 12 (UT) with the Megacam imager (McLeod et al. 2015) on the Magellan Clay telescope. Megacam has a $\sim 24^{\prime} \times 24^{\prime}$ field of view and a binned pixel scale of 0 " 16 . The seeing was $\sim 0$ " 9 and $\sim 0$ ". 6 for the $g$ - and $r$-band, respectively. We took fourteen $300 \mathrm{~s}$ exposures in the $g$-band and six $300 \mathrm{~s}$ exposures in the $r$-band, which were dithered to fill in the gaps between the CCDs. The data were reduced using the Megacam pipeline at the Smithsonian Astrophysical Observatory Telescope Data Center.

We perform point-spread function fitting photometry on each of the stacked final images, using the suite of programs DAOPHOT and ALLFRAME (Stetson 1994) and following the procedure described in Crnojević et al. (2016). To calibrate our data, we used the AAVSO Photometric All-Sky Survey (APASS; Henden et al. 2012) $g$ - and $r$-band photometry and cross-matched it with short (60 s) Eri II exposures, propagating the derived zeropoints and color terms to the longer science exposures. The calibrated catalogs were corrected for Galactic extinction (Schlegel et al. 1998; Schlafly \& Finkbeiner 2011) star by star (the average extinction across the image is $\sim 0.01 \mathrm{mag}$ ), and we present dereddened $g_{0}$ and $r_{0}$ magnitudes throughout this work.

We performed a series of artificial star tests to determine our photometric errors and completeness as a function of magnitude and color. Over many experiments, we injected $\sim 10$ times the number of artificial stars into our data as were detected in the unaltered images. The artificial stars were homogeneously distributed across the field of view, with a color-magnitude distribution similar to that of the observed 




Figure 1. Panel (a): CMD of Eri II sources within its half-light radius. We show photometric errors and 50\% completeness levels (red dashed lines) as derived from artificial star tests. Panel (b): background field CMD rescaled to the area in panel (a). Panel (c): Hess density diagram of the Eri II CMD after background subtraction. Panel (d): same as panel (c), with a $10 \mathrm{Gyr},[\mathrm{Fe} / \mathrm{H}]=-2.5$ isochrone (red line), a $3 \mathrm{Gyr},[\mathrm{Fe} / \mathrm{H}]=-2.0$ isochrone (cyan line; Dotter et al. 2008), and the $\mathrm{HB}$ fiducial of M92 (blue solid line) overplotted, all shifted to the distance of Eri II.

sources, but extending $\sim 2$ magnitudes fainter. The photometry of the images with artificial star injections was derived exactly in the same way as for the real data. The color-averaged $50 \%$ completeness limits are $r_{0} \sim 26$ and $g_{0} \sim 26.6 \mathrm{mag}$. In Figure 1, we present the CMD of Eri II, along with our derived photometric errors and 50\% completeness limits.

\section{ANALYSIS}

The CMD for sources within Eri II's half-light radius $\left(r_{h}\right)$ is presented in Figure 1, along with a background CMD derived from two regions of identical size located away from Eri II. Our CMD reaches $\sim 3$ mag deeper than the discovery data (Bechtol et al. 2015; Koposov et al. 2015).

\subsection{Stellar Populations}

Eri II is not simply a single old and metal-poor stellar population, in contrast to many of the new, faint satellites of the MW (Brown et al. 2014). The color and slope of the RGB and the blue extent of the extended horizontal branch (HB; Figure 1) are characteristic of old, metal-poor $(\gtrsim 10 \mathrm{Gyr}$, $[\mathrm{Fe} / \mathrm{H}] \lesssim-1.0)$ populations such as those in globular clusters or ultra-faint dwarfs. The HB is not purely blue, but has a red HB (RHB) portion as well: while this can be a feature of old and metal-rich populations, the RGB slope of Eri II seems to exclude the presence of significantly enriched stars. Alternatively, the RHB could arise from a metal-poor population marginally younger $(\sim 2 \mathrm{Gyr})$ than the oldest one, or as a second parameter effect (Salaris \& Cassisi 2005). The objects between the BHB and the RHB at a range of magnitudes are candidate RR Lyrae stars.

We consider the Hess diagram for the field-subtracted CMD (Figure 1): an excess of sources is present above the subgiant

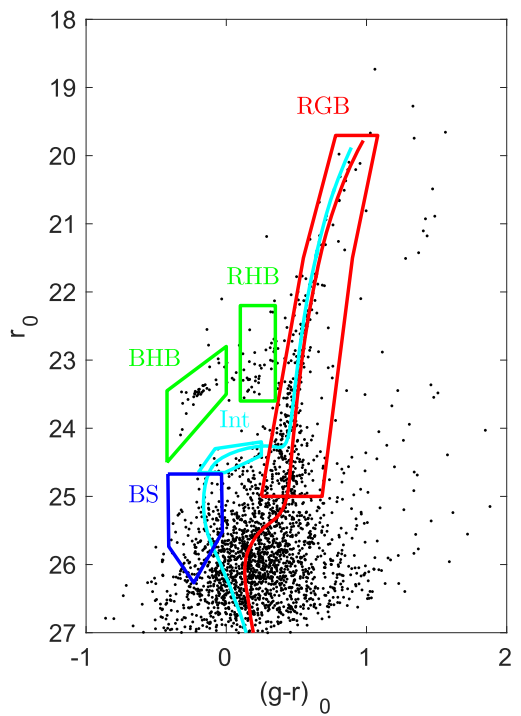

Figure 2. CMD of Eri II, showing the selection boxes used to select different subpopulations. Isochrones are as in Figure 1.

branch portion of the old isochrone, around $-0.20 \lesssim(g-r)_{0} \lesssim 0.15$ and $24.3 \lesssim r_{0} \lesssim 25.3$. This feature is well fit by a $\sim 3 \mathrm{Gyr}$ and $[\mathrm{Fe} / \mathrm{H}]=-2.0$ isochrone (panel (d) of Figure 1), or alternatively by a $\sim 4 \mathrm{Gyr}$ population as metal-poor as the old RGB. A significantly younger and more metal-rich population can be ruled out, since it would produce a RGB redder than observed; similarly, for older ages the turnoff would be too faint to match the observed one. We cannot exclude the possibility that these are, at least in part, blue straggler (BS) stars arising from binary systems, 

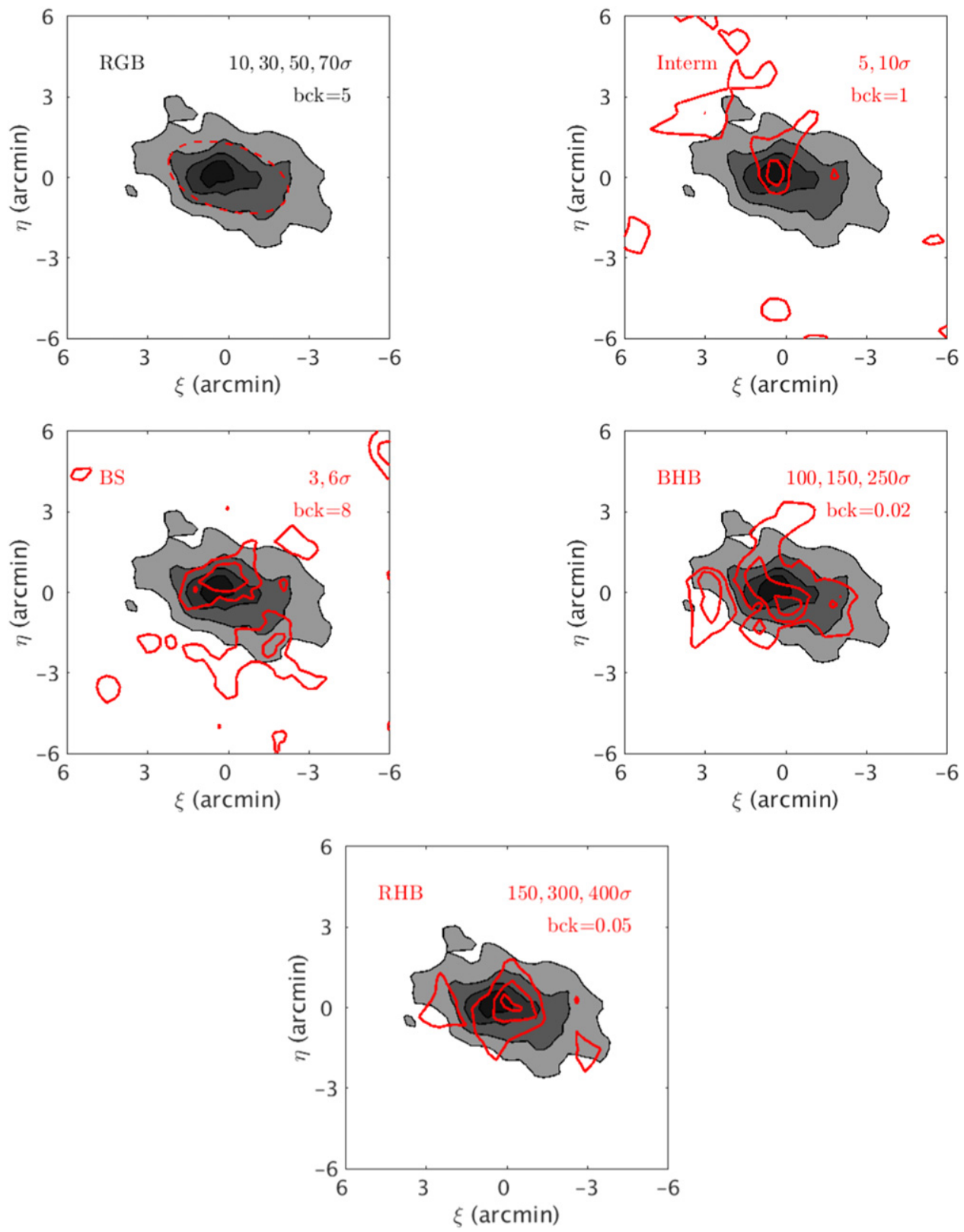

Figure 3. Stellar maps of different CMD features. The red dashed ellipse in the top left panel indicates $r_{h}$. Isodensity contours are logarithmically spaced and indicate $\sigma$ above the field level, with values reported in each subpanel; also indicated is the field level in units of stars per $\operatorname{arcmin}^{2}$. The subpopulations are: RGB stars (thin contours and filled distribution in each subpanel), intermediate-age subgiant branch stars (red contours in top right panel), candidate BS stars (red contours in central left panel), BHB stars (red contours in central right panel), and RHB stars (red contours in bottom panel).

which can mimic intermediate-age populations (for a discussion about the "blue plume" in faint MW satellites, see Sand et al. 2010; Santana et al. 2013; Weisz et al. 2016). The ratio of the number of candidate BS stars to the number of blue HB (BHB) stars (computed following Deason et al. 2015 ) is $\sim 1.7 \pm 0.4$, consistent with empirical values derived for dwarf galaxies with luminosities similar to Eri II (e.g., Deason et al. 2015). This supports the BS interpretation for these stars.

We select different CMD populations as shown in Figure 2 and draw their spatial distribution (as density maps) in Figure 3. RGB stars present a regular and elongated shape, with no clear signs of disturbance. Intermediate-age stars produce a very noisy stellar density map but have a significant overdensity at the center of Eri II. The map for candidate BS stars is similarly noisy, but it still shows a possible central concentration, which would favor the intermediate-age population interpretation (BS binary systems are expected to have a spatial distribution similar to that of the old population). The BHB and RHB maps have a very high significance above the field level; the BHB sample's highest overdensity is slightly offset from Eri II's center.

Koposov et al. (2015) suggested that Eri II possesses a young stellar population component ( $250 \mathrm{Myr})$ based on the presence of a few stars blueward of the RGB's bright end (at $r_{0} \sim 20$ and $\left.(g-r)_{0} \sim 0.2\right)$. If these were truly young stars, we would detect sources along the main-sequence portion (around $r_{0} \gtrsim 22.5$ ) of the young isochrone plotted in Figure 16 of Koposov et al. (2015), but we do not observe these in our data set. 
Table 1

Properties of Eri II and Its Cluster

\begin{tabular}{|c|c|c|}
\hline Parameter & Eri II & Cluster \\
\hline R.A. (h:m:s) & 03:44:20.1土10". 5 & $03: 44: 22.2 \pm 1^{\prime \prime}$ \\
\hline Decl. (d:m:s) & $-43: 32: 01.7 \pm 5 " ! 3$ & $-43: 31: 59.2 \pm 2^{\prime \prime}$ \\
\hline$(m-M)_{0}(\mathrm{mag})$ & $22.8 \pm 0.1$ & $\cdots$ \\
\hline $\mathrm{D}(\mathrm{kpc})$ & $366 \pm 17$ & $\cdots$ \\
\hline$\epsilon$ & $0.48 \pm 0.04$ & $\cdots$ \\
\hline $\mathrm{PA}\left(\mathrm{N}\right.$ to $\left.\mathrm{E} ;^{o}\right)$ & $72.6 \pm 3.3$ & $\cdots$ \\
\hline$r_{h}(\operatorname{arcmin})$ & $2.31 \pm 0.12$ & $0.11 \pm 0.01$ \\
\hline$r_{h}(\mathrm{pc})$ & $277 \pm 14$ & $13 \pm 1$ \\
\hline$n$ (Sérsic index) & $1^{\mathrm{a}}$ & $0.19 \pm 0.05$ \\
\hline$\mu_{V, 0}\left(\operatorname{mag} \operatorname{arcsec}^{-2}\right)$ & $27.2 \pm 0.3$ & $25.7 \pm 0.2$ \\
\hline$M_{V}(\mathrm{mag})$ & $-7.1 \pm 0.3$ & $-3.5 \pm 0.6$ \\
\hline$\left\langle(g-r)_{0}\right\rangle(\mathrm{mag})$ & $0.5 \pm 0.3$ & $0.4 \pm 0.2$ \\
\hline$M_{\mathrm{HI}} / L_{V}\left(M_{\odot} / L_{\odot}\right)$ & $<0.036$ & $\cdots$ \\
\hline
\end{tabular}

Note.

${ }^{a}$ An exponential profile was assumed for Eri II.

\subsection{Distance}

We rederive the distance to Eri II from the luminosity of its BHB (e.g., Sand et al. 2012). We adopt the fiducial HB sequence for M92 from Bernard et al. (2014) and convert the Pan-STARRS1 magnitudes into the SDSS system following Tonry et al. (2012). While we adopt the nominal M92 distance value reported by Bernard et al. (2014; $(m-M)=14.65$, from Harris 2010), other studies have measured slightly different values, as high as $(m-M)=14.74$ (see di Cecco et al. 2010), but they are all within their respective $1 \sigma$ errors. We thus adopt an M92 distance uncertainty of $\sigma_{(m-M), M 92}=0.1$, which is the major source of error in our measurement. We perform a least-squares fitting of the M92 HB fiducial to Eri II's BHB, and we find a best-fit value of $(m-M)_{0}=22.8 \pm 0.1$, corresponding to a distance of $D=366 \pm 17 \mathrm{Mpc}$. This is consistent with the results of Koposov et al. (2015).

\subsection{Structural Properties and Luminosity}

We re-determine structural parameters for Eri II with the same maximum likelihood technique utilized for most of the other Local Group (LG) dwarfs (Martin et al. 2008; Sand et al. 2012). RGB stars were included in our structural analysis; we did not explicitly include the $\mathrm{HB}$ or potential younger stellar population, although any true younger stellar population would inevitably have RGB stars that would also be included. We did not mask the central star cluster for our structural analysis, although a separate calculation with the star cluster masked out led to structural parameters within $1 \sigma$ of the original. The free parameters of our exponential profile plus constant background fit were: central position, position angle, ellipticity, $r_{h}$, and background surface density (results are reported in Table 1). Uncertainties were determined by bootstrap resampling the data 1000 times and recalculating the structural parameters for each resample.

Eri II's surface brightness profile is well fit by an exponential with $r_{h}=277 \pm 14 \mathrm{pc}$, and its ellipticity is high $(\epsilon=0.48 \pm 0.04$ ), similar to other faint MW satellites (Sand et al. 2012). The central surface brightness is derived from the total luminosity (see below), $r_{h}$, and the ellipticity, and it is $\mu_{V, 0}=27.2 \pm 0.3 \mathrm{mag} \operatorname{arcsec}^{-2}$. While most of the derived parameters are in line with the discovery results, our $r_{h}$ is higher by a factor of $\sim 1.5$ : deep follow-up photometric studies often lead to increased $r_{h}$ values with respect to shallower data sets (e.g., Crnojević et al. 2014).

Given the likely presence of two subpopulations, we derive the absolute magnitude of Eri II as follows. We derive the completeness-corrected and field-subtracted luminosity for each subpopulation by summing the flux of stars in a magnitude range where the corresponding isochrones do not overlap within the observational errors $\left(24.7<r_{0}<25.5\right.$ for the intermediate-age population and $24.4<r_{0}<25.5$ for the older population). The luminosity is computed within $r_{h}$ and then multiplied by two. We extrapolate the total luminosity given by each component starting from the fraction of light contributed in the selected magnitude ranges, as estimated from theoretical luminosity functions from Dotter et al. (2008). These are constructed with a Salpeter initial mass function, one for a $10 \mathrm{Gyr},[\mathrm{Fe} / \mathrm{H}]=-2.5$ population and the other for a $3 \mathrm{Gyr},[\mathrm{Fe} / \mathrm{H}]=-2.0$ population. The luminosities associated with the subpopulations are $L_{V \text {,old }} \sim 5.6 \pm 1.5 \times 10^{4} L_{\odot}$ and $L_{V \text {,int }} \sim 3.5 \pm 3.0 \times 10^{3} L_{\odot}:$ the intermediate-age population contributes $\sim 6 \%$ of Eri II's light, and only a few percent of its stellar mass. The total absolute magnitude for Eri II is $M_{V}=-7.1 \pm 0.3$ (the $V$-band magnitude was obtained using the Jester et al. 2005 transformations), which is between the values reported by Bechtol et al. (2015) and Koposov et al. (2015). If the stars brighter than the old subgiant branch had a BS component, the absolute magnitude of Eri II would not significantly change, given their small number.

\subsection{HI Gas Content}

To constrain the HI content of Eri II, we obtained positionswitched HI observations (AGBT-16A-186; PI: Spekkens) on the Robert C. Byrd Green Bank Telescope (GBT) on 2016 February 23. In the ranges $-500 \leqslant V_{L S R K} \leqslant-50 \mathrm{~km} \mathrm{~s}^{-1}$ and $50 \leqslant V_{L S R K} \leqslant 500 \mathrm{~km} \mathrm{~s}^{-1}$ _encompassing possible HI recessional velocities of MW satellites that do not overlap with the MW HI emission itself along the Eri II line of sight- the GBT spectrum has an rms noise $\sigma=1.2 \mathrm{mJy}$ at a spectral resolution of $15 \mathrm{~km} \mathrm{~s}^{-1}$. We do not find any $\mathrm{HI}$ emission in this velocity range within the FWHM $=9 ! 1=960 \mathrm{pc}$ GBT beam at this frequency. Combining this non-detection with the distance and luminosity values derived above, a putative $\mathrm{HI}$ counterpart has a $5 \sigma, 15 \mathrm{~km} \mathrm{~s}^{-1} \mathrm{HI}$ mass upper limit of $M_{\mathrm{HI}}^{\lim }=2.8 \times 10^{3} M_{\odot}$ out to $\sim 1.5 r_{h}$, and therefore $M_{\mathrm{HI}} / L_{V}<0.036 M_{\odot} / L_{\odot}$; this constraint is a factor of $\sim 7$ more stringent than that derived by Westmeier et al. (2015) using HIPASS data. Eri II is therefore extremely gas-poor, similar to other dwarf spheroidal galaxies in the Local Volume (Grcevich \& Putman 2009; Spekkens et al. 2014).

\subsection{The Central Star Cluster}

A dense collection of stars near Eri II's center stand out from the overall diffuse nature of the dwarf (Figure 4; see also Koposov et al. 2015). This likely star cluster makes Eri II, at $M_{V} \sim-7.1 \mathrm{mag}$, by far the least luminous dwarf galaxy to host such an object. The position of the cluster is nearly at the calculated center of Eri II ( $\sim 45$ pc off-center in projection) and within its uncertainties (see Table 1). Small offsets between centrally located clusters and the exact center of their parent dwarf galaxy are commonly observed (e.g., Georgiev 

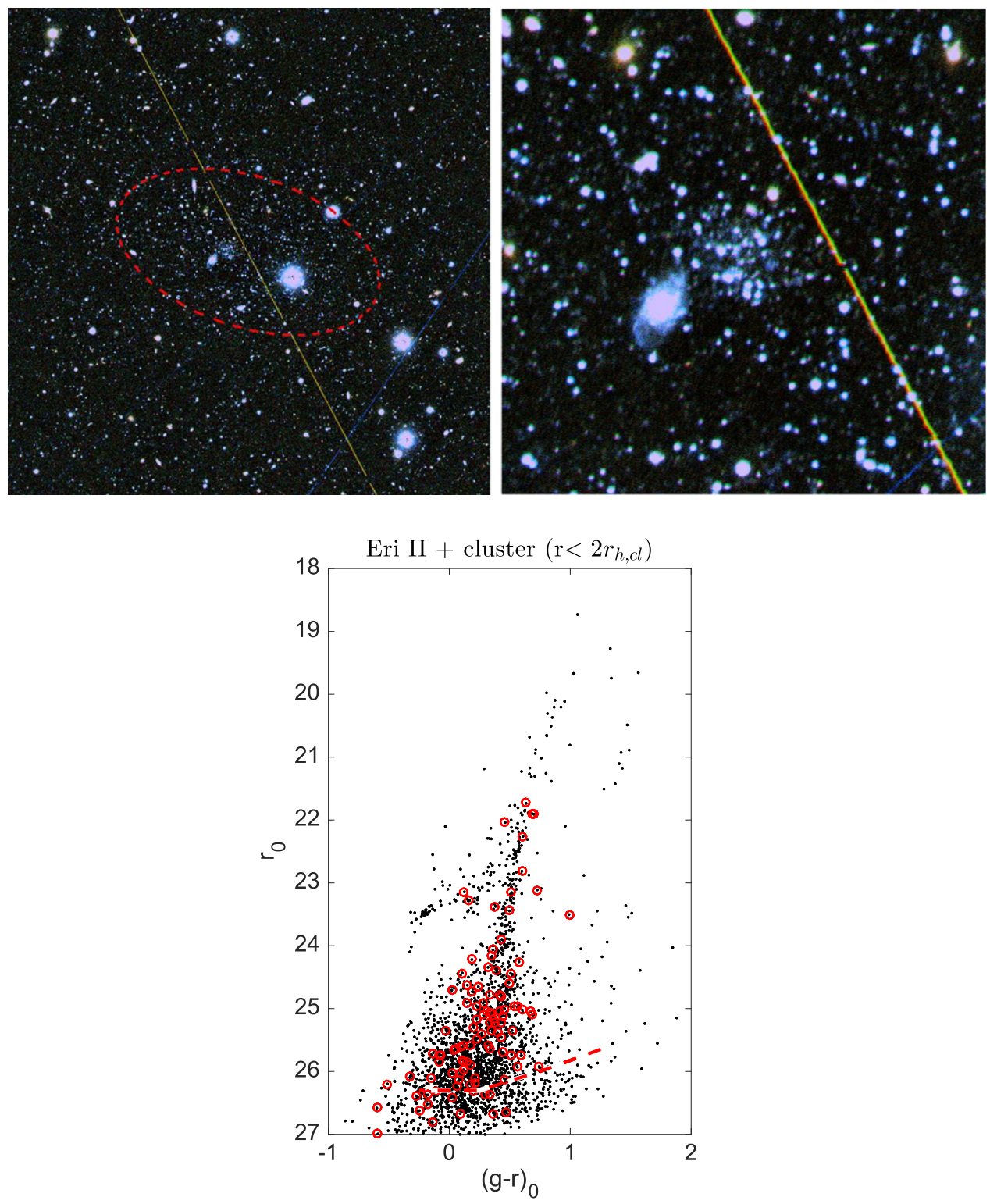

Figure 4. RGB-color images of Eri II. A satellite trail is apparent in both panels. Upper left panel: $10^{\prime} \times 10^{\prime}$ field centered on Eri II; a red dashed ellipse indicates $r_{h}$. Upper right panel: $2^{\prime} \times 2^{\prime}$ zoom-in on Eri II's cluster, which is partially resolved into stars. Lower panel: dereddened CMD for Eri II within $r_{h}$; in red we overlay stars from the cluster within twice its computed $r_{h}$.

et al. 2009), so we plausibly consider this as a candidate nuclear star cluster, more specifically the only one known within such a faint galaxy.

In Figure 4, we overlay on Eri II's CMD the stars contained within twice the $r_{h}$ of its cluster (see below). The center of the cluster was computed iteratively as the average of the stellar positions within circles of decreasing radius. Since the cluster is only partially resolved, its CMD is poorly populated, but still consistent with Eri II's CMD. To test this, we randomly draw 1000 sub-CMDs with the same number of sources as in the cluster from Eri II's CMD: the results resemble the cluster's $\mathrm{CMD}$, and $\sim 15 \%$ of the realizations also lack a BHB. We compute the cluster's properties via integrated photometry, assuming a circular radius (the cluster is visually round). The surface brightness profile for the cluster is derived after masking bright stars and background galaxies and is then fit with a Sérsic profile (best-fit values are reported in Table 1). The absolute magnitude is derived by integrating the best-fit
Sérsic profile and is $M_{V}=-3.5 \pm 0.6$ at the distance of Eri II, which contributes to $\sim 4 \%$ of its host's luminosity. We discuss the properties of the cluster in the next section.

\section{DISCUSSION AND CONCLUSIONS}

We have presented deep Magellan/Megacam photometric follow-up observations of Eri II, and derived improved distance, structural properties, and luminosity measurements. At a distance of $370 \mathrm{kpc}$, Eri II is just beyond the estimated MW virial radius, where most dwarfs show signs of neutral gas/recent star formation. However, Eri II is not currently forming stars (based on the lack of a blue main sequence), unlike the two gas-rich dwarfs found at a similar Galactocentric distance, Leo T $\left(M_{V} \sim-8.0\right.$; e.g., Weisz et al. 2012) and Phoenix $\left(M_{V} \sim-10.0\right.$; e.g., Battaglia et al. 2012). Our followup GBT observations reveal that Eri II is extremely gas-poor. This suggests that it is not on first infall and may have already 
interacted with the MW. The stellar distribution shows no signs of disruption down to the current photometric depth, disfavoring tidal interactions. Rather, the stellar and gas properties of Eri II are consistent with a ram pressure stripping scenario in which its gas reservoir was swept away by the MW's coronal gas during a previous pericentric passage (e.g., Gatto et al. 2013).

At $M_{V}=-7.1$, Eri II is the faintest dwarf galaxy known to host a stellar cluster, consistent with being at the center of its host. Compared to the LG's globular clusters, Eri II's cluster would be one of the faintest objects at its $r_{h}$ value, and its absolute magnitude and $r_{h}$ place it in a similar parameter space to the least luminous and most compact MW ultra-faint dwarfs. With $r_{h}=13 \mathrm{pc}$, it can be regarded as an "extended" cluster: these are only observed at large galactocentric distances in M31, or in dwarf galaxies like NGC 6822 and Scl-dE1 (see Da Costa et al. 2009; Mackey et al. 2010). Such objects are believed to survive only within weak tidal fields (e.g., Hurley \& Mackey 2010).

Is Eri II's cluster a survivor? We compute its half-mass relaxation time based on our derived luminosity, assuming a mass-to-light ratio of $\sim 1.5$, and obtain $\sim 2$ Gyr (e.g., Da Costa et al. 2009). Since the timescale for evaporation due to twobody relaxation is on the order of $\gtrsim 10$ times this quantity, internal processes must not be relevant for our object, and the weak tidal field of Eri II likely does not contribute to a possible tidal truncation. A disruption could happen in the presence of strong external tidal forces; however, the lack of tidal features in Eri II and its large Galactocentric distance make this an unrealistic possibility.

Nuclear star clusters in dwarf galaxies likely form via inspiral due to dynamical friction, rather than being born in situ (see, e.g., den Brok et al. 2014), even though this interpretation is far from definitive. Around the MW and M31 only a handful of satellites host clusters, the faintest being Fornax $\left(M_{V} \sim-13.5\right)$ and And I $\left(M_{V} \sim-11.7\right.$; McConnachie 2012), but none of these seem to be a nuclear star cluster. In Fornax, the presence of a globular cluster system and the lack of a nuclear cluster suggest that the dynamical friction timescale is prolonged by the existence of a cored, rather than cuspy, dark matter profile (Goerdt et al. 2006). Beyond the LG, a few dwarfs as faint as $M_{V} \sim-10$ host candidate globular clusters (Da Costa et al. 2009; Georgiev et al. 2009); however, a deep study of the Fornax galaxy cluster found no galaxies with $M_{i}>-10$ (out of a sample of $\sim 60$ ) with nuclear star clusters (Muñoz et al. 2015). Clearly, the cluster in Eri II is a rare event: this will be key to investigate the formation of clusters in such faint galaxies, and the modeling of its dynamical friction timescales may help constrain Eri II's dark matter profile.

Why, finally, are there no other dwarfs fainter than $M_{V} \sim-10$ with confirmed clusters? Zaritsky et al. (2016) suggest a scenario where at least a fraction of the outer halo Galactic clusters may in fact reside in extremely low stellar density subhalos (like Eri II), which have so far gone undetected. This uncharted territory certainly deserves further exploration and detailed modeling.

We thank the anonymous referee for their thoughtful suggestions and Tom Maccarone for useful discussions. D.J. S. acknowledges financial support from NSF grant AST1412504; D.Z. acknowledges support from NSF AST-
1311326; B.W. acknowledges support from NSF AST1151462. This paper uses data products produced by the OIR Telescope Data Center, supported by the Smithsonian Astrophysical Observatory. The National Radio Astronomy operates the GBT and is a facility of the NSF operated under cooperative agreement by Associated Universities, Inc.

\section{REFERENCES}

Battaglia, G., Rejkuba, M., Tolstoy, E., Irwin, M. J., \& Beccari, G. 2012, MNRAS, 424, 1113

Bechtol, K., Drlica-Wagner, A., Balbinot, E., et al. 2015, ApJ, 807, 50

Belokurov, V., Irwin, M. J., Koposov, S. E., et al. 2014, MNRAS, 441, 2124

Bernard, E. J., Ferguson, A. M. N., Schlafly, E. F., et al. 2014, MNRAS, 442, 2999

Brown, T. M., Tumlinson, J., Geha, M., et al. 2014, ApJ, 796, 91

Crnojević, D., Ferguson, A. M. N., Irwin, M. J., et al. 2014, MNRAS, 445,3862

Crnojević, D., Sand, D. J., Spekkens, K., et al. 2016, ApJ, 823, 19

Da Costa, G. S., Grebel, E. K., Jerjen, H., Rejkuba, M., \& Sharina, M. E. 2009, AJ, 137, 4361

Deason, A. J., Belokurov, V., \& Weisz, D. R. 2015, MNRAS, 448, L77

de Jong, J. T. A., Harris, J., Coleman, M. G., et al. 2008, ApJ, 680, 1112

den Brok, M., Peletier, R. F., Seth, A., et al. 2014, MNRAS, 445, 2385

di Cecco, A., Becucci, R., Bono, G., et al. 2010, PASP, 122, 991

Dotter, A., Chaboyer, B., Jevremović, D., et al. 2008, ApJS, 178, 89

Drlica-Wagner, A., Bechtol, K., Rykoff, E. S., et al. 2015, ApJ, 813, 109

Gatto, A., Fraternali, F., Read, J. I., et al. 2013, MNRAS, 433, 2749

Georgiev, I. Y., Hilker, M., Puzia, T. H., Goudfrooij, P., \& Baumgardt, H. 2009, MNRAS, 396, 1075

Goerdt, T., Moore, B., Read, J. I., Stadel, J., \& Zemp, M. 2006, MNRAS, 368, 1073

Grcevich, J., \& Putman, M. E. 2009, ApJ, 696, 385

Harris, W. E. 2010, arXiv:1012.3224

Henden, A. A., Levine, S. E., Terrell, D., Smith, T. C., \& Welch, D. 2012, JAVSO, 40, 430

Hurley, J. R., \& Mackey, A. D. 2010, MNRAS, 408, 2353

Jester, S., Schneider, D. P., Richards, G. T., et al. 2005, AJ, 130, 873

Kim, D., \& Jerjen, H. 2015, ApJL, 808, L39

Kim, D., Jerjen, H., Mackey, D., Da Costa, G. S., \& Milone, A. P. 2015, ApJL, 804, L44

Klypin, A., Zhao, H., \& Somerville, R. S. 2002, ApJ, 573, 597

Koposov, S. E., Belokurov, V., Torrealba, G., \& Evans, N. W. 2015, ApJ, 805,130

Laevens, B. P. M., Martin, N. F., Ibata, R. A., et al. 2015a, ApJL, 802, L18 Laevens, B. P. M., Martin, N. F., Bernard, E. J., et al. 2015b, ApJ, 813, 44

Mackey, A. D., Huxor, A. P., Ferguson, A. M. N., et al. 2010, ApJL, 717, L11

Martin, N. F., de Jong, J. T. A., \& Rix, H.-W. 2008, ApJ, 684, 1075

Martin, N. F., Nidever, D. L., Besla, G., et al. 2015, ApJL, 804, L5

McConnachie, A. W. 2012, AJ, 144, 4

McLeod, B., Geary, J., Conroy, M., et al. 2015, PASP, 127, 366

Muñoz, R. P., Eigenthaler, P., Puzia, T. H., et al. 2015, ApJL, 813, L15

Ryan-Weber, E. V., Begum, A., Oosterloo, T., et al. 2008, MNRAS, 384, 535

Salaris, M., \& Cassisi, S. 2005, Evolution of Stars and Stellar Populations, ed. M. Salaris, \& S. Cassisi (Weinheim: Wiley-VCH)

Sand, D. J., Seth, A., Olszewski, E. W., et al. 2010, ApJ, 718, 530

Sand, D. J., Strader, J., Willman, B., et al. 2012, ApJ, 756, 79

Santana, F. A., Muñoz, R. R., Geha, M., et al. 2013, ApJ, 774, 106

Schlafly, E. F., \& Finkbeiner, D. P. 2011, ApJ, 737, 103

Schlegel, D. J., Finkbeiner, D. P., \& Davis, M. 1998, ApJ, 500, 525

Spekkens, K., Urbancic, N., Mason, B. S., Willman, B., \& Aguirre, J. E. 2014 ApJL, 795, L5

Stetson, P. B. 1994, PASP, 106, 250

Tonry, J. L., Stubbs, C. W., Lykke, K. R., et al. 2012, ApJ, 750, 99

Torrealba, G., Koposov, S. E., Belokurov, V., \& Irwin, M. 2016, MNRAS, 459, 2370

Weisz, D. R., Zucker, D. B., Dolphin, A. E., et al. 2012, ApJ, 748, 88

Weisz, D. R., Koposov, S. E., Dolphin, A. E., et al. 2016, ApJ, 822, 32

Westmeier, T., Staveley-Smith, L., Calabretta, M., et al. 2015, MNRAS, 453,338

Zaritsky, D., Crnojević, D., \& Sand, D. J. 2016, ApJL, in press (arXiv:1604.08594) 